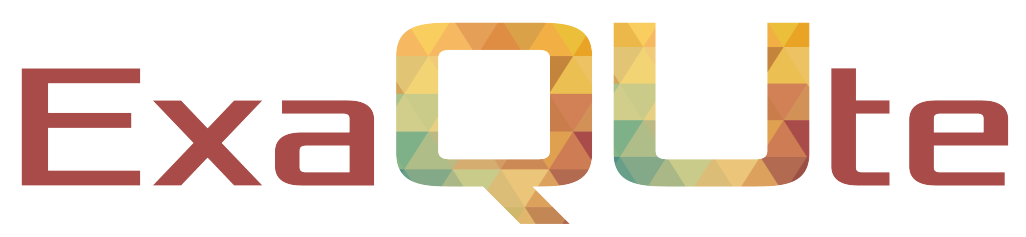

Exascale Quantification of Uncertainties for

Technology and Science Simulation

\title{
D2.1 Meshing "stub" implementation of the capabilities to be delivered
}

\section{Document information table}

\begin{tabular}{|l|l|}
\hline Contract number: & 800898 \\
\hline Project acronym: & ExaQUte \\
\hline Project Coordinator: & CIMNE \\
\hline Document Responsible Partner: & INRIA \\
\hline Deliverable Type: & Report, Other \\
\hline Dissemination Level: & PUblic \\
\hline Related WP \& Task: & WP 2 Task 2.1 \\
\hline Status: & Final Version \\
\hline
\end{tabular}

\begin{tabular}{|ll|}
\hline${ }^{\star \star}{ }^{\star}$ & This project has received funding from \\
${ }^{\star}$ & the European Union's Horizon 2020 \\
${ }_{\star}^{\star} \star \star$ & research and innovation programme \\
under grant agreement No 800898
\end{tabular}




\section{Authoring}

\begin{tabular}{|l|l|l|l|l|}
\hline \multicolumn{5}{|l|}{ Prepared by: } \\
\hline Authors & Partner & Modified Page/Sections & Version & Comments \\
\hline Algiane Froehly & INRIA & & & \\
\hline Vicente Mataix & CIMNE & & & \\
\hline Carlos Roig & CIMNE & & & \\
\hline Contributors & & & & \\
\hline & & & & \\
\hline & & & & \\
\hline
\end{tabular}

\section{Change Log}

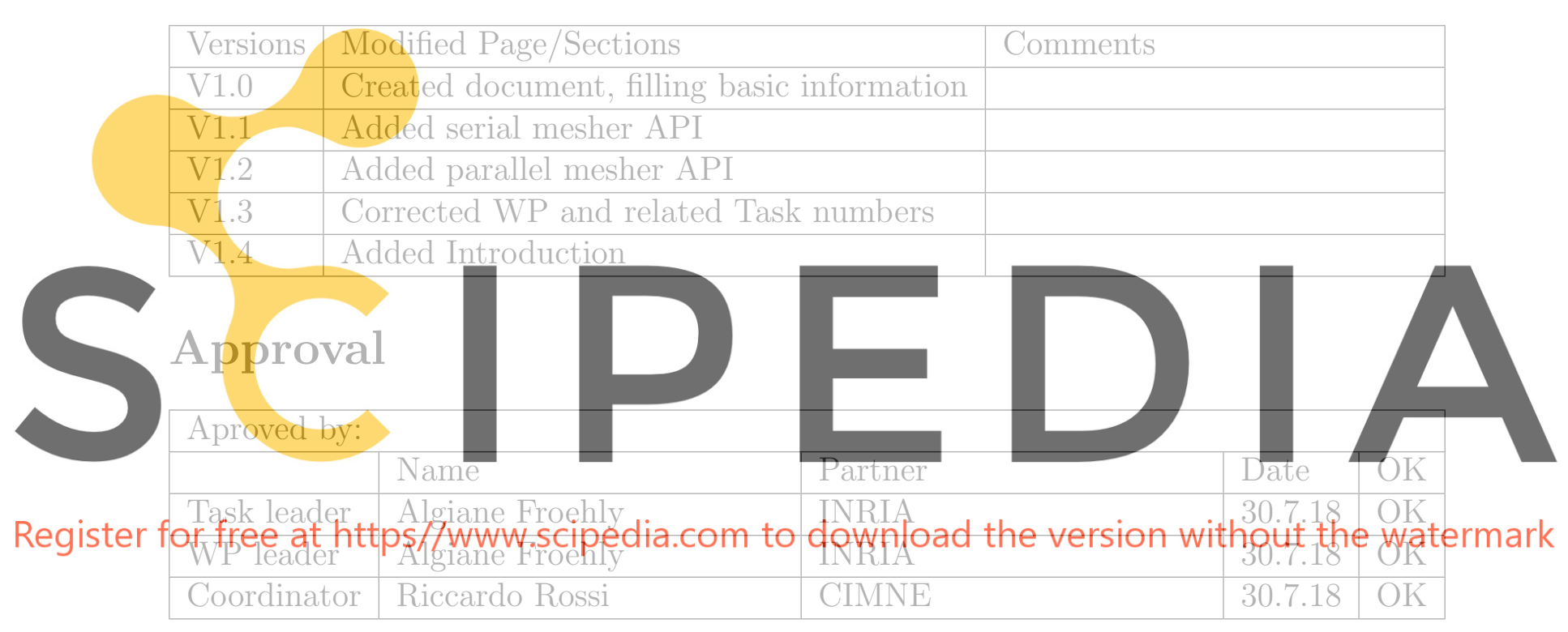




\section{Executive summary}

This document presents a description of the MMG interface adapted to Kratos as well as its parallel counterpart with ParMMG which will be implemented in task 2.1. In the description are included the following items:

- Description of the requirements of the interfaces;

- Description of the data structures used both in Kratos and MMG;

- Proposal of an initial interface for ParMMG.

- The input needed for the model;

- The definition of the model system matrices;

- The solver and time marching schemes;

- The results obtained, and the link to the 3D detailed model.
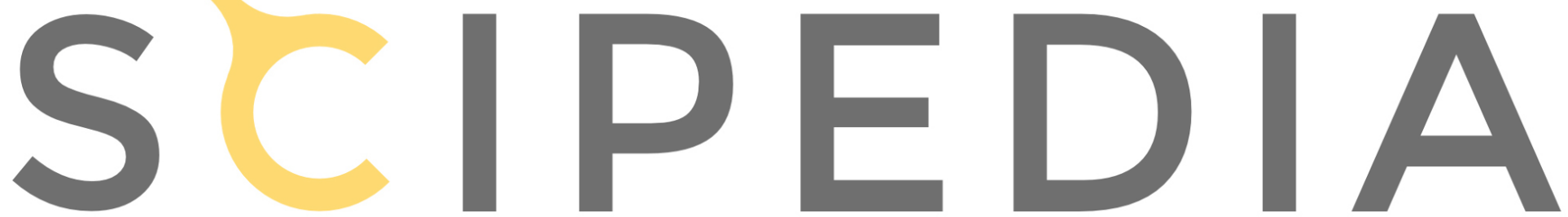

Register for free at https//www.scipedia.com to download the version without the watermark 


\section{Table of contents}

1 Introduction $\quad 8$

2 Interface Description: existing $M M G$ interface $\quad 8$

2.1 InitMesh . . . . . . . . . . . . . . . . . . . 8

2.2 InitializeMeshData . . . . . . . . . . . . . . . 8

2.3 InitializeSolData . . . . . . . . . . . . . . . . . . . 10

2.4 CheckMeshData . . . . . . . . . . . . . . . . . 10

2.5 ExecuteRemeshing . . . . . . . . . . . . . . . . . 10

3 Interface Proposal: ParMMG interface 12

3.1 InitMesh . . . . . . . . . . . . . . . . . . . . . . . 12

3.2 InitializeMeshData. . . . . . . . . . . . . . . . . . . . . . . . . . 12

3.3 InitializeSolData

3.4 ExecuteRemeshing
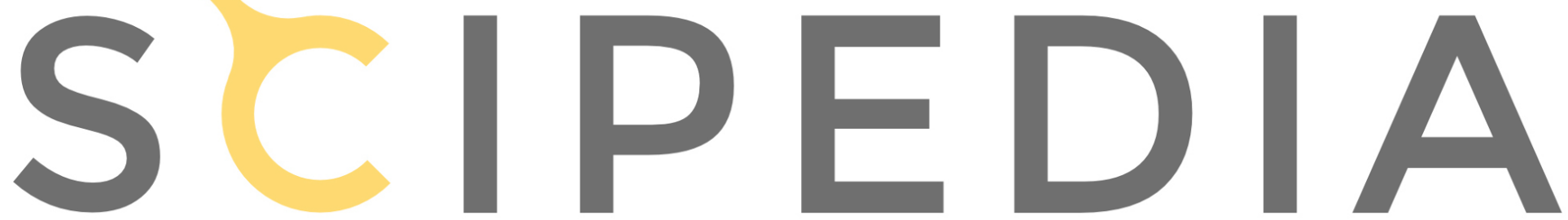

Register for free at https//www.scipedia.com to download the version without the watermark 


\section{List of Figures}

1 MMGProcess Initialization . . . . . . . . . . . . . . . . . . . . . 9

2 Submodelpart used in order to set BC . . . . . . . . . . . . . . . . 9

3 Colour submodelpart creation . . . . . . . . . . . . . . . . . 10

4 MMGProcess Initialize Mesh Data . . . . . . . . . . . . . . . . . 17

$5 \quad$ MMGProcess Initialize Solution Data . . . . . . . . . . . . . . . 18

$6 \quad$ MMGProcess Check . . . . . . . . . . . . . . . . . . . . 18

$7 \quad$ MMGProcess Remesh . . . . . . . . . . . . . . . . . . . . . 19

$8 \quad$ MMGProcess Finalize . . . . . . . . . . . . . . . . . . . . . . . 19

9 ParMMGProcess Initialization . . . . . . . . . . . . . . . . . . . . 20

10 ParMMGProcess Initialize Mesh Data . . . . . . . . . . . . . . . . 21

11 ParMMGProcess Initialize Metric Data . . . . . . . . . . . . . . . . . . 22

12 ParMMGProcess Remesh . . . . . . . . . . . . . . . . . . . 22

13 ParMMGProcess Finalize . . . . . . . . . . . . . . . . . . . 23
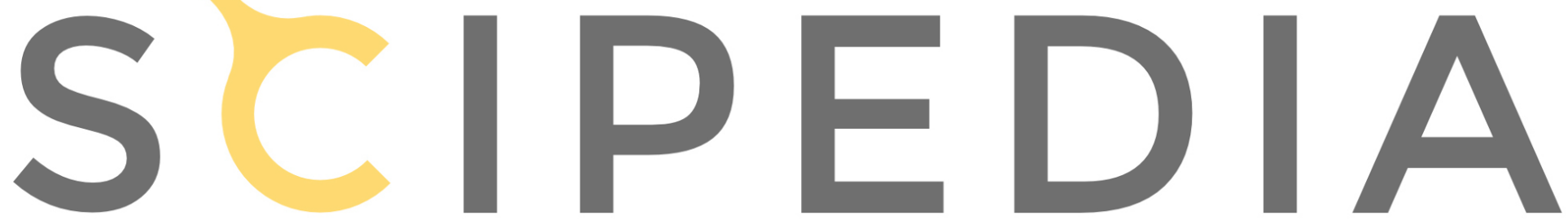

Register for free at https//www.scipedia.com to download the version without the watermark 


\section{List of Tables}

1 Nomenclature / Acronym list . . . . . . . . . . . . 7

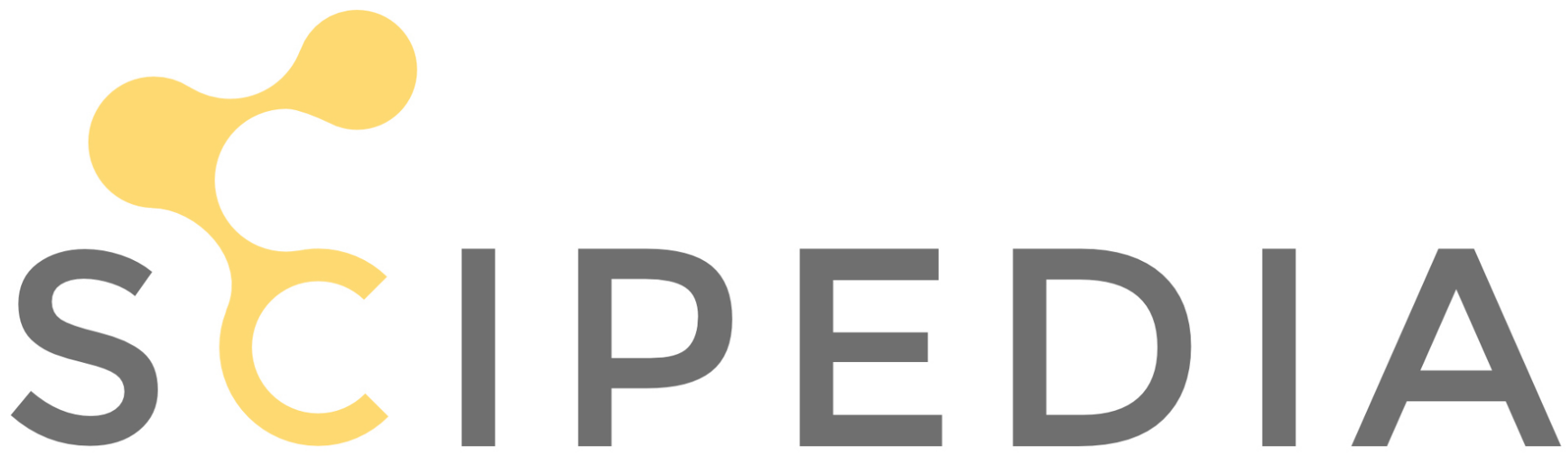

Register for free at https//www.scipedia.com to download the version without the watermark 


\section{Nomenclature / Acronym list}

\begin{tabular}{|l|l|}
\hline Acronym & Meaning \\
\hline API & Application Programming Interface \\
\hline OOP & Object Oriented Programming \\
\hline BC & Boundary Condition \\
\hline MPI & Message Pasing Interface \\
\hline GP & Gauss Point \\
\hline
\end{tabular}

Table 1: Nomenclature / Acronym list
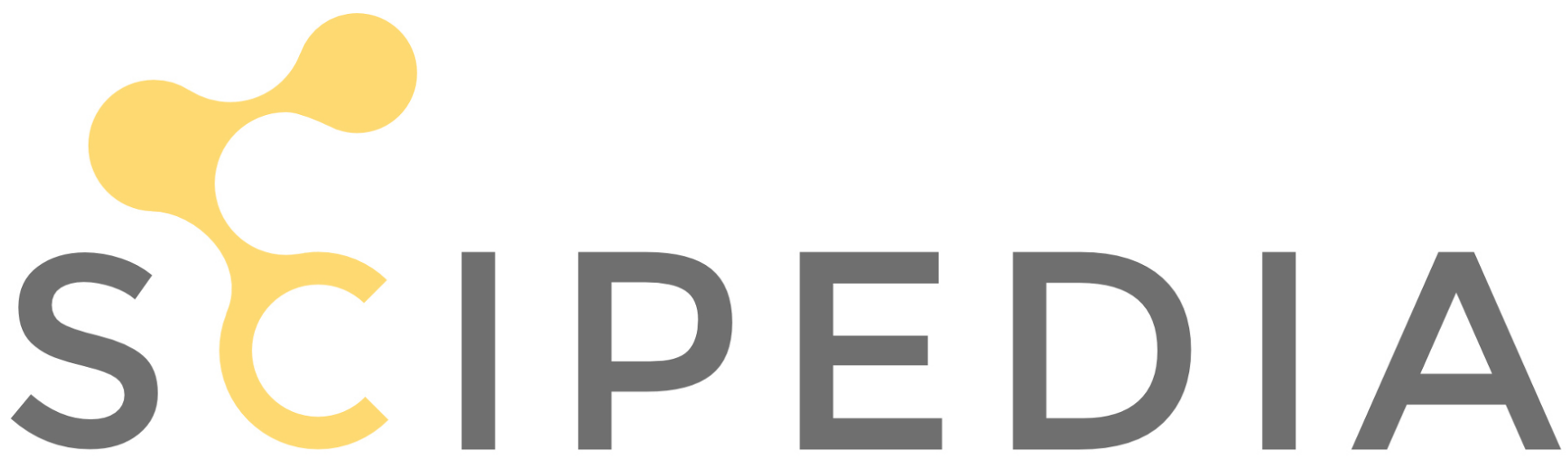

Register for free at https//www.scipedia.com to download the version without the watermark 


\section{Introduction}

This deliverable describes the status of the current implementation of Kratos Mulitphysics [1] and MMG [2] [3] interface to provide an API which will allow to use its meshing capabilities as described in the WP2. Also, we define the first iteration of the future interface for parallel meshing based on ParMMG.

\section{Interface Description: existing $M M G$ interface}

The way the interface will be exposed to Kratos Multiphysics is via a remeshing process that will be placed inside Kratos own meshing application (MeshingApplication) called MMGProcess. This process will prepare the input modelpart data and will convert the model into $M M G$ format. This conversion will be done using several call to $M M G$ exposed methods which are directly wrapped inside Kratos.

This Kratos process considers as input the modelpart of the problem we are interested in remesh and the configuration parameters that has been read from a .json file. This gives us a lot of flexibilty, because the parameters can be changed dinamically without refactoring, so adding functionalities to the process is easier than in a coventional input design.

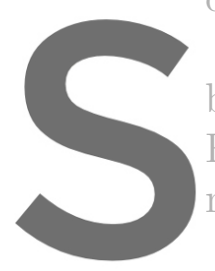

It is important between $2 \mathrm{D}$ and $3 \mathrm{D}$

From the point of viev

made in such a way

The proposed work
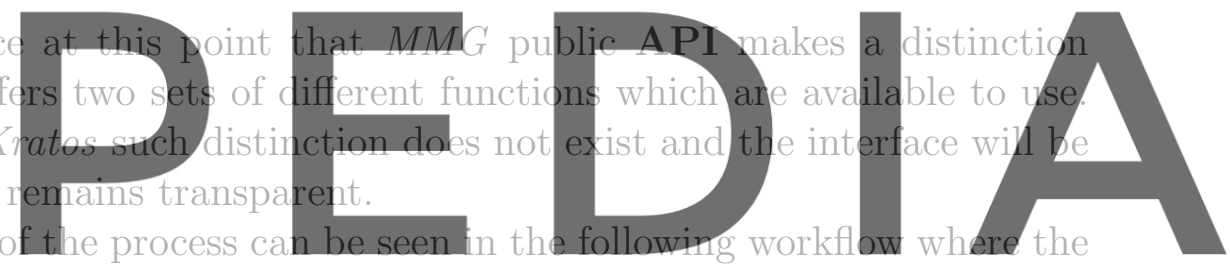

specific calls to $M M G$ are displayed.

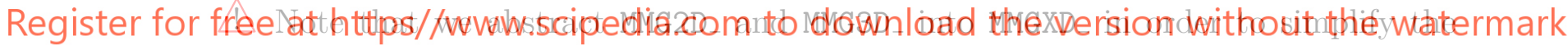
diagram.

$\triangle$ Note also that MMGXD_Set_iparameter and MMGXD_Set_dparameter (for integer and doubles) are also abstracted to MMGXD_Set_Xparameter for similar reasons.

\subsection{InitMesh}

As its name indciates it will serve as the init function and directly call the init function of $M M G$. This will create and initialize the pointers of the mesh and the solution used bt used by $M M G$. This calls MMGXD_Init mesh from the $M M G$ API (see Figure 1).

\subsection{InitializeMeshData}

\section{Auxiliar sub model part creation}

We will start with the creation of auxiliar sub model parts for flags. These model parts are created in order to preserve the flags of the nodes, elements and conditions after remesh. These auxiliar submodelparts will be removed after remesh.

\section{Colour mapping}

After that, we will follow with the creation of a colour map, or model part list. Here we will compute a map that will serve us in order tor preserve Kratos sub-modelpart structure. In in our implementations we use a process to set the BC (both Neumann or 


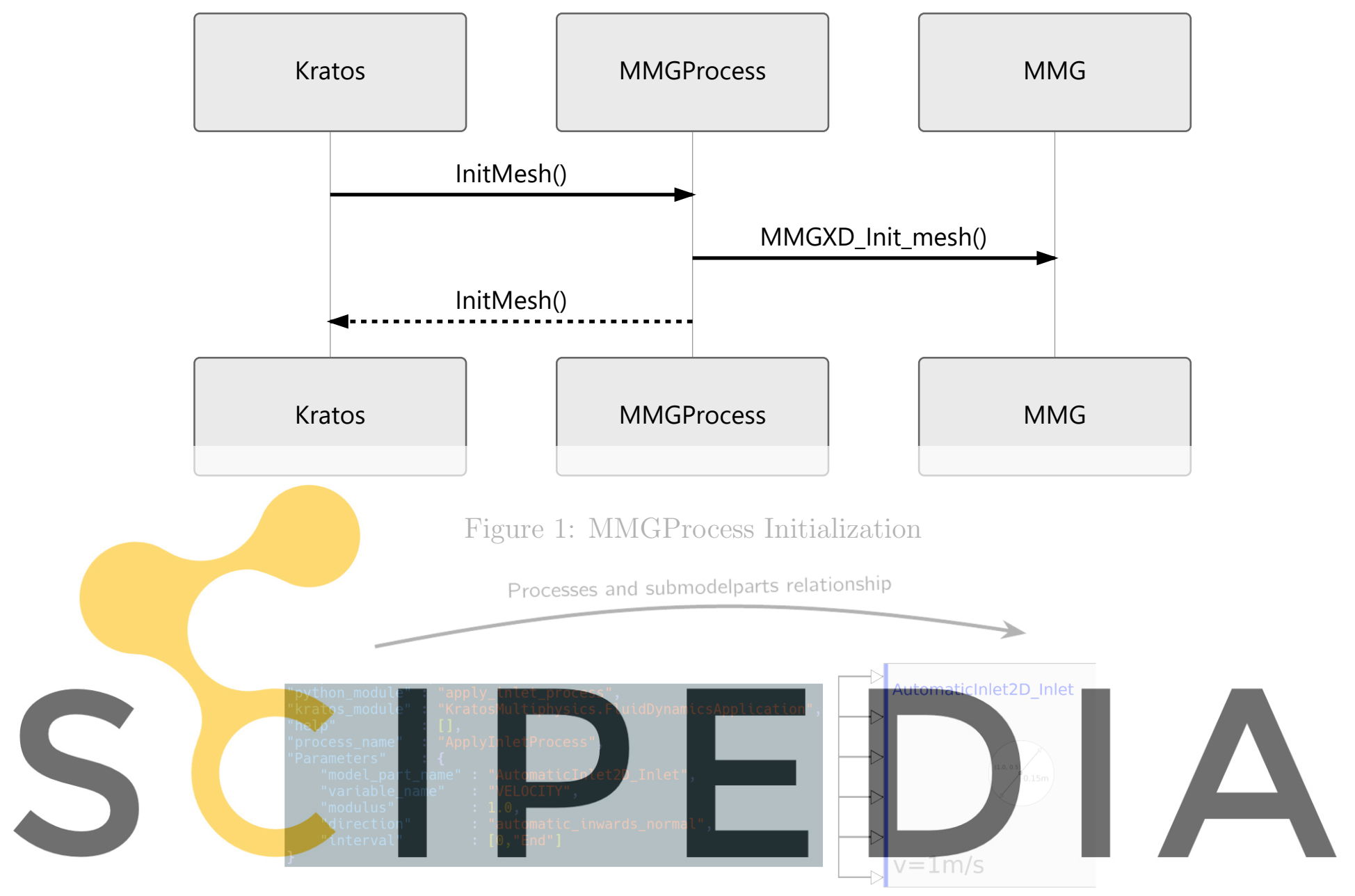

Register for free at https//www.scipedia.com to download the version without the watermark Figure 2: Submodelpart used in order to set BC

Dirichlet). We identify the parts where to set this BC with the use of submodelparts (see Figure 2). The same component (node, condition, element) can belong to different submodelparts, so if we want to have an unique ids for each component we need to compute the common belonging to each component. We call this colour see Figure 3.

\section{Node preservation}

At this stage, if we want to preseve a part of the mesh we have the option to block individual nodes using the MMGXD_Set_requiredVertex function of $M M G$.

\section{Transfer mesh data to $M M G$}

In order to be able of doing that we will compute first the number of total nodes, elements and conditions in order to allocate the memory necessary to remesh. Also, we will convert Kratos nodes to $M M G$ vertices and finally gemoetries in Kratos to geometries in MMG which will be mapped to edges, triangles, prism and tetrahedras depending on the source geometry (see Figure 4).

- Kratos_Line2D2 $\rightarrow$ MMG Edge (MMG2D_Set_edge);

- Kratos_Line3D2 $\rightarrow$ MMG Edge (MMG3D_Set_edge); 


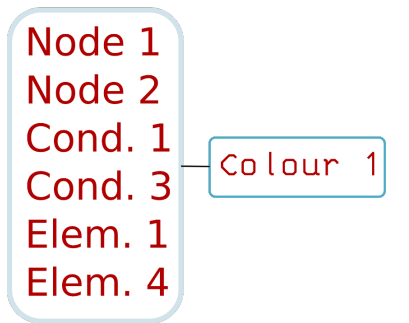

Node 1

Node 2

Cond. 1

Cond. 3

Elem. 4

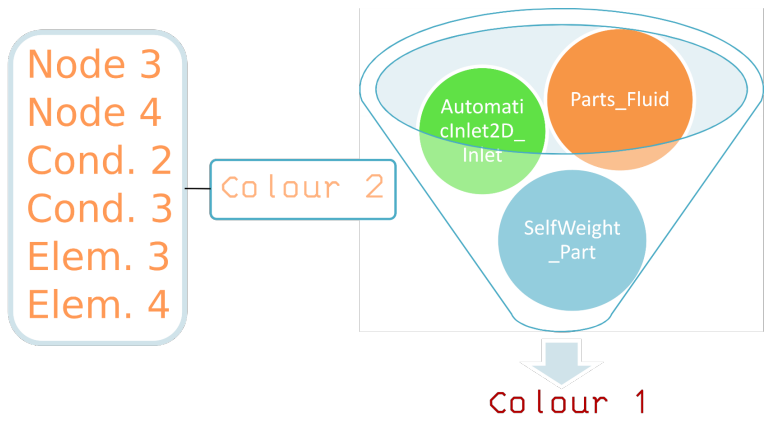

Figure 3: Colour submodelpart creation

- Kratos_Triangle3D3 $\rightarrow$ MMG Triangle (MMG3D_Set_triangle);

- Kratos_Quadrialeteral3D4 $\rightarrow$ MMG Quadrilateral (MMG3D_Set_quadrilateral);

- Kratos_Tetrahedra3D4 $\rightarrow$ MMG Tetrahedron (MMG3D_Set_tetrahedron);

- Kratos_Prism3D6 $\rightarrow$ MMG Prism (MMG3D_Set_prism);

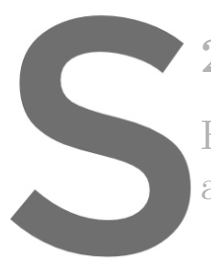

2.3 InitializeSolDa.ta
Here we allocate the metric. We always use
allthoug scalar wrapper also exists.
This is done in two differents steps, first
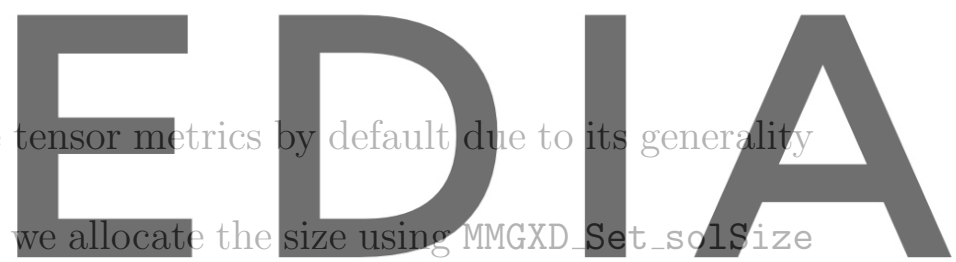

and then setting its values using MMGXD_Set_tensorSol, MMG3D_Set_vectorSol and MMGXD_Set_SolSize

Register foffreeg be lattps//www.scipedia.com to download the version without the watermark

\subsection{CheckMieshData}

Before proceed with the remeshing we call the routines from $M M G$ designed in order to check that the information transfered to the API of $M M G$ is consistent, this is the method MMGXD_Chk_meshData(). See Figure 6.

\subsection{ExecuteRemeshing}

We call the remeshing process as well as setting the general configuration values in mmg. At detail, the list of parameters set in this step are the following ${ }^{1}$ :

- MMGXD_DPARAM_hausd : The Hausdorff controls the smothness of the boundary;

- MMGXD_IPARAM_nomove: This parameters sets if we allow to avoid or allow the point recolocation;

- MMGXD_IPARAM_nosurf: This parameters sets if we don't allow the surfaces modification;

- MMGXD_IPARAM_noinsert: This parameters sets if we don't insert nodes on mesh;

\footnotetext{
${ }^{1}$ These parameters are registered as enums
} 
- MMGXD_IPARAM_noswap: This parameters sets if we don't swap the mesh;

- MMGXD_IPARAM_angle: This parameters is used in order to deactivate/activate the angle detection;

- MMGXD_DPARAM_hgrad: This parameter is used in order to set the gradation;

- MMGXD_DPARAM_hmin: This parameters sets the minimum size of the mesh;

- MMGXD_DPARAM_hmax: This parameters sets the maximum size of the mesh.

And finally we call the remeshing method MMGXD_mmgXdlib() as seen in Figure 7.

After executing the remesh, the new nodes, conditions and elements are created and transfered to the corresponding submodelparts using the respective colours previously computed, which correspond with the $M M G$ reference.

\section{Save output mesh and solution}

In case we are interested in getting the mesh and solution obtained in the .mesh and . sol file of $M M G$ it exists the possibility to export the new mesh and solution to external files with the use of SaveSolutionToFile(). It calls the MMGXD_Set_outputMeshName and MMGXD_Set_outputSolName methods form the $M M G$ API to set the filenames and the MMGXD_saveMesh and MMGXD_saveSol methods to write the output files.

\section{FreeMemory}

After we have computed all the relative to $M M G$ we can now release the memory relative to it. We use the MMGXD_Free_all() method from the API for that pourpose. See Figure 8 for more details.

\section{ReorderAllIds}

During this step the ids of the element, conditions and nodes are reordered. So the ids will vary from 1 to $\mathrm{n}$, being $\mathrm{n}$ the number of each component. This will not affect to the problem itself, due to the OOP nature of Kratos, if the ids are reordered, it will be reorder in all the objects depending of it.

This step must be done before and after remesh in order to avoid problematics with the remeshing process. Some steps of the process, including call to MMG, could do some assumptions about ordered ids of the different containers of the problem. Then the best way to avoid these problematics will be to reorder the ids, following the previously exposed methodology.

\section{Interpolation of mesh information}

This step is necessary in order to preserve the information of the old mesh in the new mesh. We use different processes and utilities in Kratos to interpolate both nodeal values and integration values from the old mesh to the new mesh. In order to interpolate internal variables the following procedures are available:

- Closest Point Transfer: It transfer the values from the closest GP;

- Least-Square projection Transfer: It transfers from the closest GP from the old mesh; 
- Shape Function Transfer: It transfer GP values to the nodes in the old mesh and then interpolate to the new mesh using the shape functions all the time.

\section{InitializeElementsAndConditions}

Finally, the created elements and conditions are initialised in order to be usable in Kratos.

\section{Interface Proposal: ParMMG interface}

We propose to update the exiting MMGProcess process of Kratos and to couple KratosMultiphysics and ParMMG in the same way as the KratosMultiphysics-MMG coupling:

- MeshingApplication $($ Kratos $) \leftarrow$ MMGProcess $($ KratosMultiphys. $) \leftarrow$ MMG API.

- The parameters and geometry of the problem are given as input for Kratos in a .json file.

This section describes the workflow for the Kratos-ParMMG coupling.

\subsection{InitMesh}

It is the method used to initialize the MMGProcess process. It calls the initialization function of ParMMG (PMMG_Init_mesh) that creates the pointer toward the main structure of ParMMG (a ParMesh) and initializes its fields:

- the mesh dimension (unlike $M M G$, ParMMG doesn't make distinction between 2D and $3 \mathrm{D}$, thus it is needed to know the mesh dimension in order to call the suitable $M M G$ functions for the $M M G$ structures initialization);

- a pointer toward a $M M G$ mesh;

- a pointer toward a $M M G$ metric;

- a pointer toward an array of $M M G$ solutions (if needed);

- the MPI communicator in which we will work.

See Figure 9 for the diagramm of the MMGProcess initialization.

\subsection{InitializeMeshData}

\section{Auxiliar sub model part creation}

This part is similar to the creation of the sub model part with $M M G$.

\section{Node preservation}

The PMMG_Set_requiredVertex function of ParMMG allows to block individual nodes and to preserve a part of the mesh. 


\section{Transfer mesh data to ParMMG}

On each processor, we will need to transfer the local mesh to ParMMG.

Again, we will need to compute first the number of nodes, elements and conditions to be able to allocate the memory for the remesher. We set the mesh size calling the PMMG_Set_meshSize function. Then, the Kratos mesh is converted into a ParMMG mesh using the following map:

- Kratos_Line2D2 or Kratos_Line3D2 $\rightarrow$ PMMG Edge (PMMG_Set_edge);

- Kratos_Triangle3D3 $\rightarrow$ PMMG Triangle (PMMG_Set_triangle);

- Kratos_Quadrialeteral3D4 $\rightarrow$ PMMG Quadrilateral (PMMG_Set_quadrilateral);

- Kratos_Tetrahedra3D4 $\rightarrow$ PMMG Tetrahedron (PMMG_Set_tetrahedron);

- Kratos_Prism3D6 $\rightarrow$ PMMG prism (PMMG_Set_prism);

Additionnaly, must provide the communication data to ParMMG. Note that ParMMG uses the partition of the dual graph of the mesh (partition of the mesh elements) while Kratos uses a partition of the mesh (partition of both the mesh elements and nodes). It leads to have more communication informations in Kratos than in ParMMG (ghost nodes). For this preliminary version of the interface specification, we will only deal with the element partition (the element partition is given to ParMMG that gives back the new element partition, leaving to Kratos the responsability of the nodes partition).

To set the communication data from Kratos to ParMMG we need:

- the local to global mapping of the nodes and elements using the PMMG_Set_loc2GlobVertices and PMMG_Set_loc2GlobTetrahedra;

- for each couple of adjacent processors, the processors IDs and the list of the nodes at their interface (PMMG_Set_interfaceNodes).

\subsection{InitializeSolData}

As ParMMG modifies both the mesh and its distribution among the processors, it must performs the solutions interpolation over the new mesh. It implies to give the solutions to $\operatorname{ParMMG}$.

Thus, the InitializeSolData process allocates now the metric and the solutions structure (Figure 11).

\section{Metric allocation}

We allocate the metric structure given its size (PMMG_Set_metSize), then we set the metric values using PMMG_Set_tensorMet.

\section{Solutions array allocation}

We allocate the array of solution structure given the number of solutions per entity and the type of each solution (scalar, vectorial or tensorial) using the PMMG_Set_allSolsSizes function. Then we set each solution value using the PMMG_Set_ithSol_inAllSols function 


\subsection{ExecuteRemeshing}

The ExecuteRemeshing process sets the ParMMG parameters and run the ParMMG library. The list of parameters setted in this step are the following ${ }^{2}$. Lot of them are similar to the $M M G$ arguments:

- PMMG_DPARAM_hausd : The Hausdorff controls the smothness of the boundary;

- PMMG_IPARAM_nomove: This parameters sets if we allow to avoid or allow the point recolocation;

- PMMG_IPARAM_nosurf: This parameters sets if we don't allow the surfaces modification;

- PMMG_IPARAM_noinsert: This parameters sets if we don't insert nodes on mesh;

- PMMG_IPARAM_noswap: This parameters sets if we don't swap the mesh;

- PMMG_IPARAM_angle: This parameters is used in order to deactivate/activate the angle detection;

- PMMG_DPARAM_hgrad: This parameter is used in order to set the gradation;

- PMMG_DPARAM_hmin: This parameters sets the minimum size of the mesh;

- PMMG_DPARAM_hmax: This parameters sets the maximum size of the mesh.

Because of the mesh repartitionning, the interpolation of the solutions from the old mesh into the new one must be done in ParMMG.

The interpolation method is choosen depending on the PMMG_IPARAM_interp argument and the wanted method:

- PMMG_INTERP_linear: linear interpolation of the solution from the old mesh in the new one;

- PMMG_INTERP_closestPoint: transfer of the solution from the closest GP.

Last we call the remeshing method PMMG_parmmglib_distributed() as seen in Figure 12.

After the remeshing step, the new nodes, conditions and elements are created and transfered to the corresponding submodelparts using the respective colours previously computed, which correspond with the $P M M G$ reference.

\section{Save output mesh, metric and solutions}

We can save the mesh, metric and solutions at .mesh and .sol format (PMMG format) using the SaveSolutionToFile() process. It calls the PMMG_Set_outputMeshName, PMMG_Set_outputMetName and PMMG_Set_outputSolsName methods form the PMMG API to set the filenames and the PMMG_saveMesh, PMMG_saveMet and PMMG_saveAllSols methods to write the output files. 


\section{FreeMemory}

We release the ParMMG memory calling the PMMG_Free_all() method. See Figure 13 for more details.

ReorderAllIds The only reordering needed from Kratos is before remeshing in order to avoid potential problematics. After remesh Kratos will take the ids provided by $\operatorname{ParMMG}$ which already computes an internal reordering.

\section{InitializeElementsAndConditions}

Finally, the created elements and conditions are initialised in order to be usable in Kratos.

\footnotetext{
${ }^{2}$ These parameters are registered as enums
} 


\section{References}

[1] P. Dadvand, J. Mora, C. González, A. Arráez, P. Ubach, and E. Oñate. Kratos: an object-oriented environment for development of multi-physics analysis software. In World Congress on Computational Mechanics, pages 485-485, Jul 2002.

[2] C. Dapogny, C. Dobrzynski, and P. Frey. Three-dimensional adaptive domain remeshing, implicit domain meshing, and applications to free and moving boundary problems. Technical report, Mar. 2013. URL https://hal.sorbonne-universite.fr/ hal-00804636.

[3] C. Dobrzynski and P. Frey. Anisotropic delaunay mesh adaptation for unsteady simulations. In Proceedings of the 17th international Meshing Roundtable, pages 177-194. Springer, 2008. 


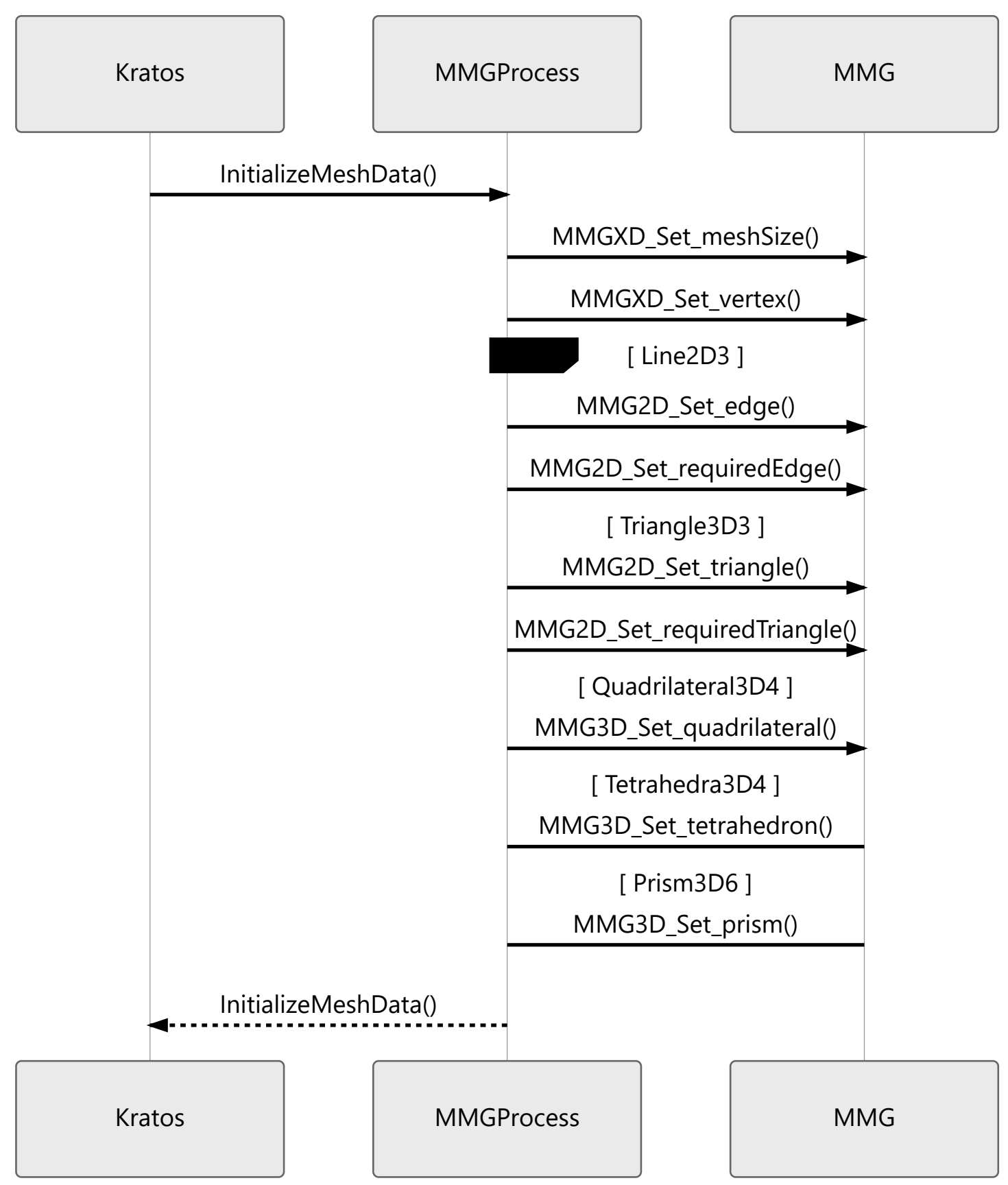

Figure 4: MMGProcess Initialize Mesh Data 


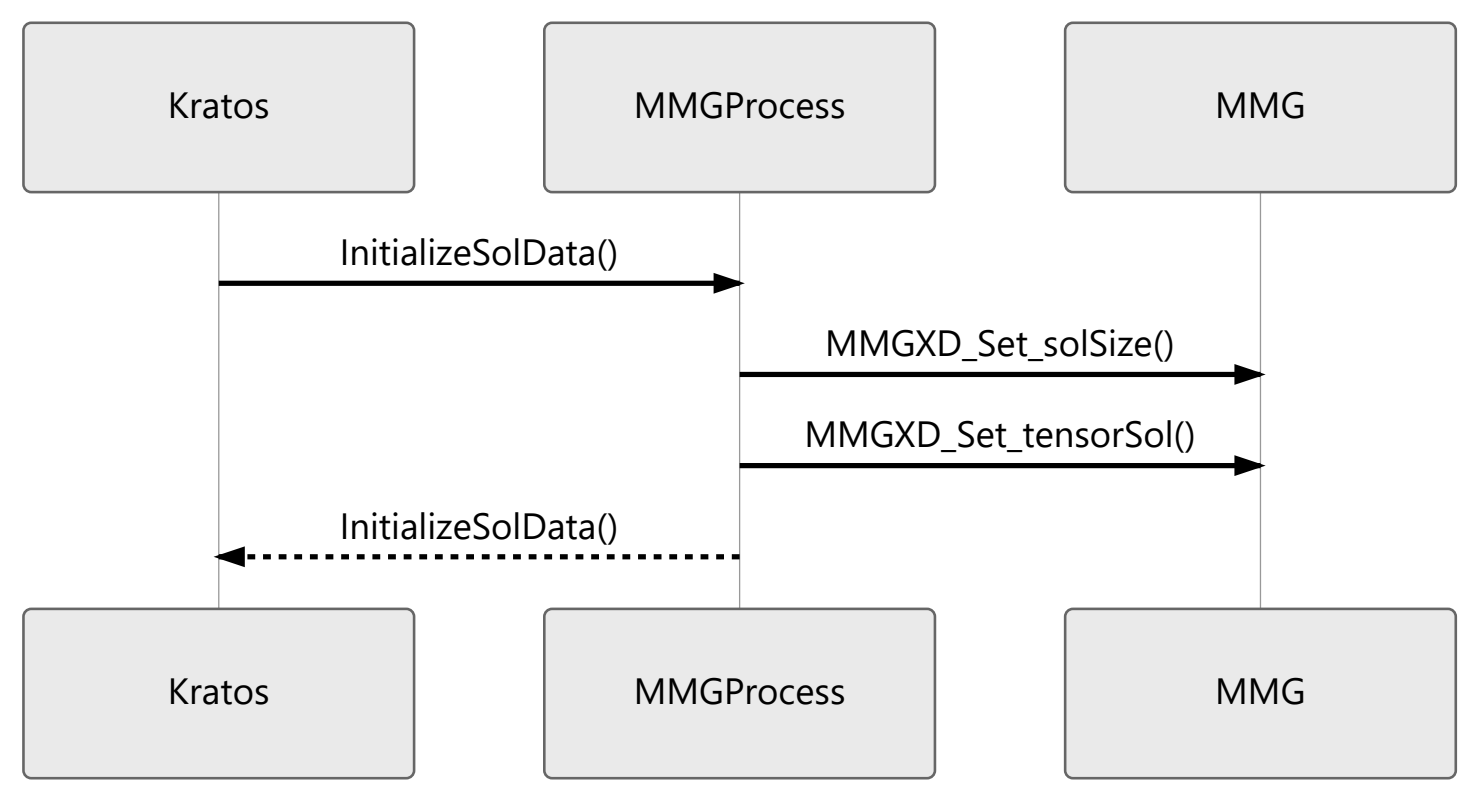

Figure 5: MMGProcess Initialize Solution Data

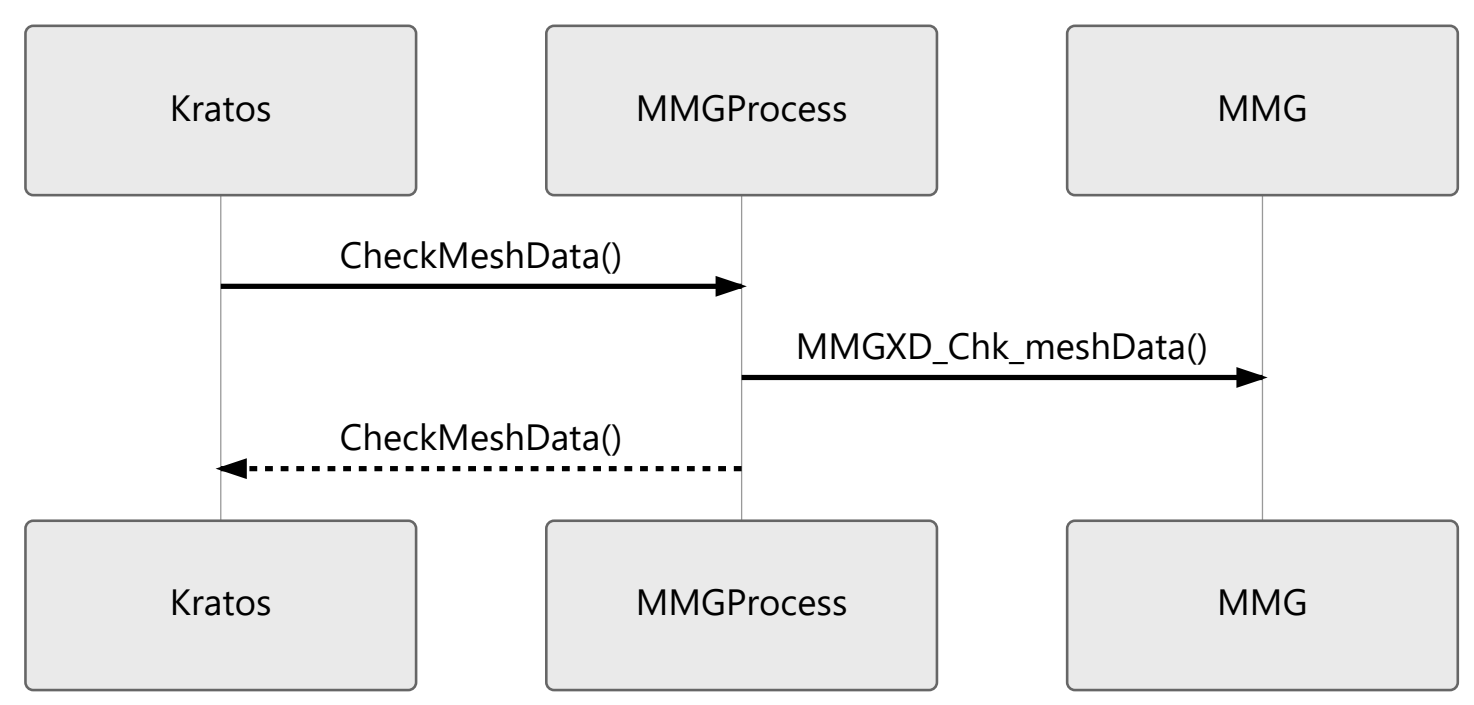

Figure 6: MMGProcess Check 


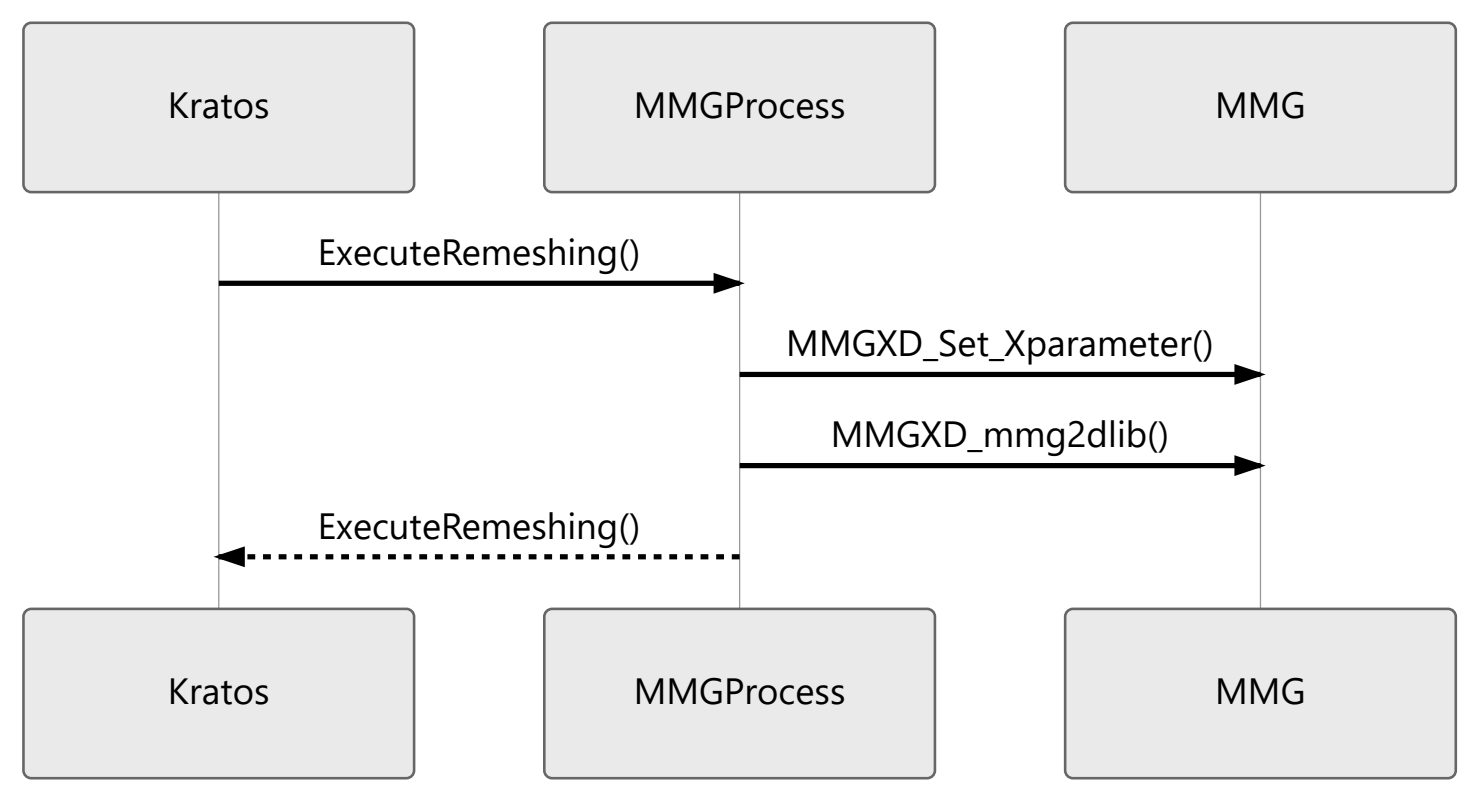

Figure 7: MMGProcess Remesh

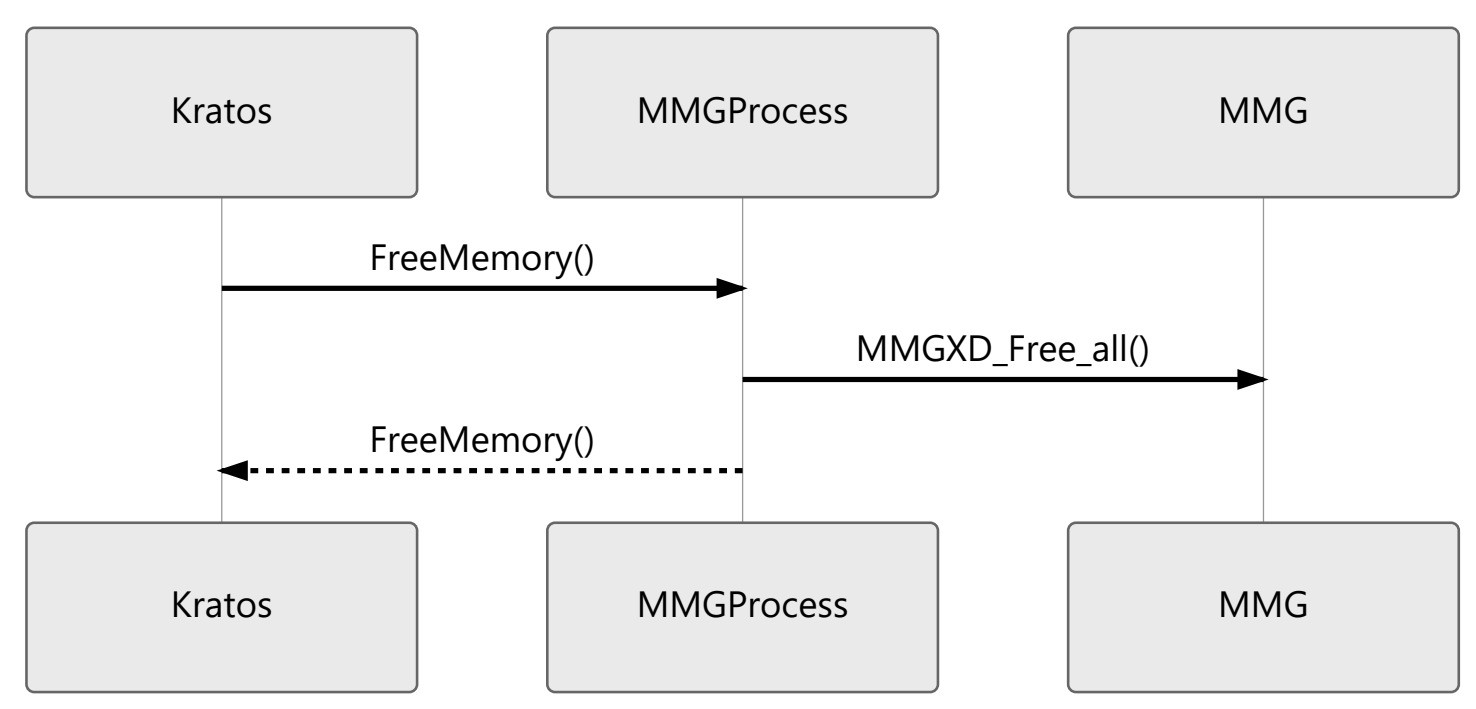

Figure 8: MMGProcess Finalize 


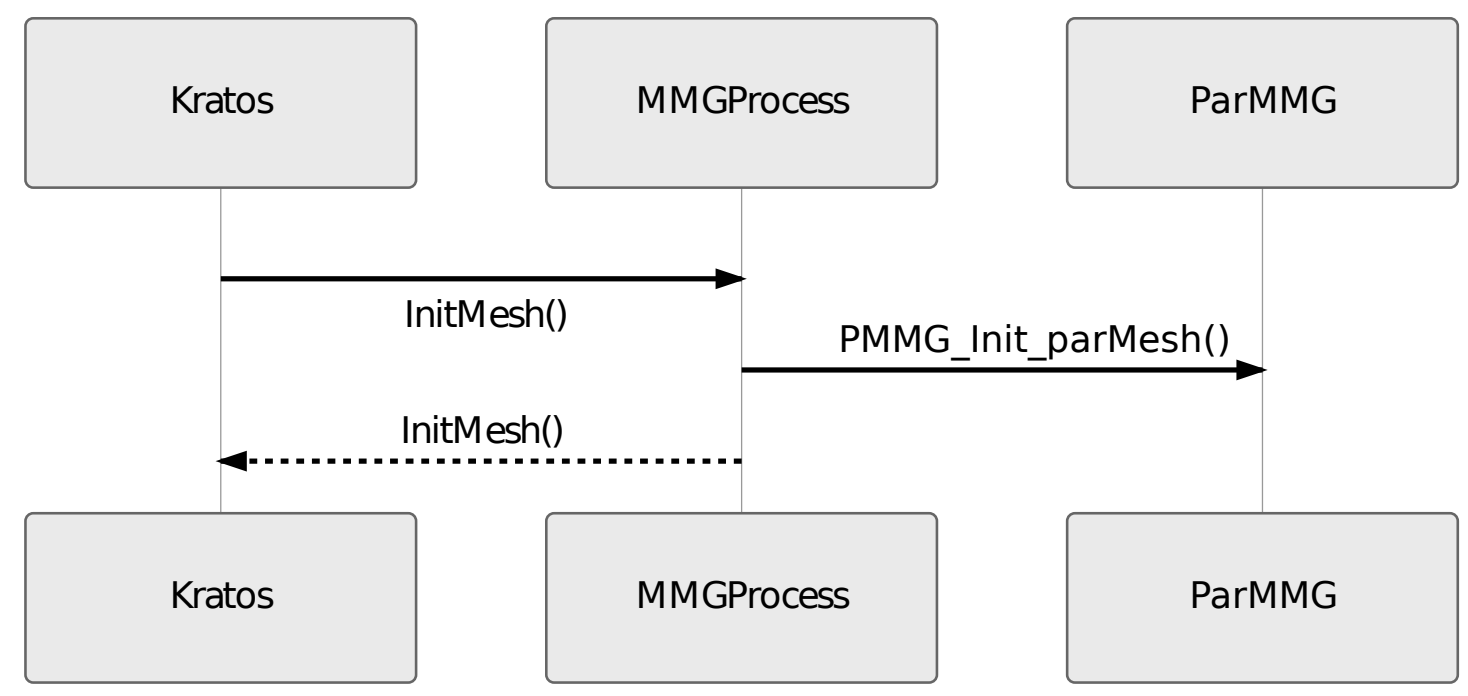

Figure 9: ParMMGProcess Initialization 


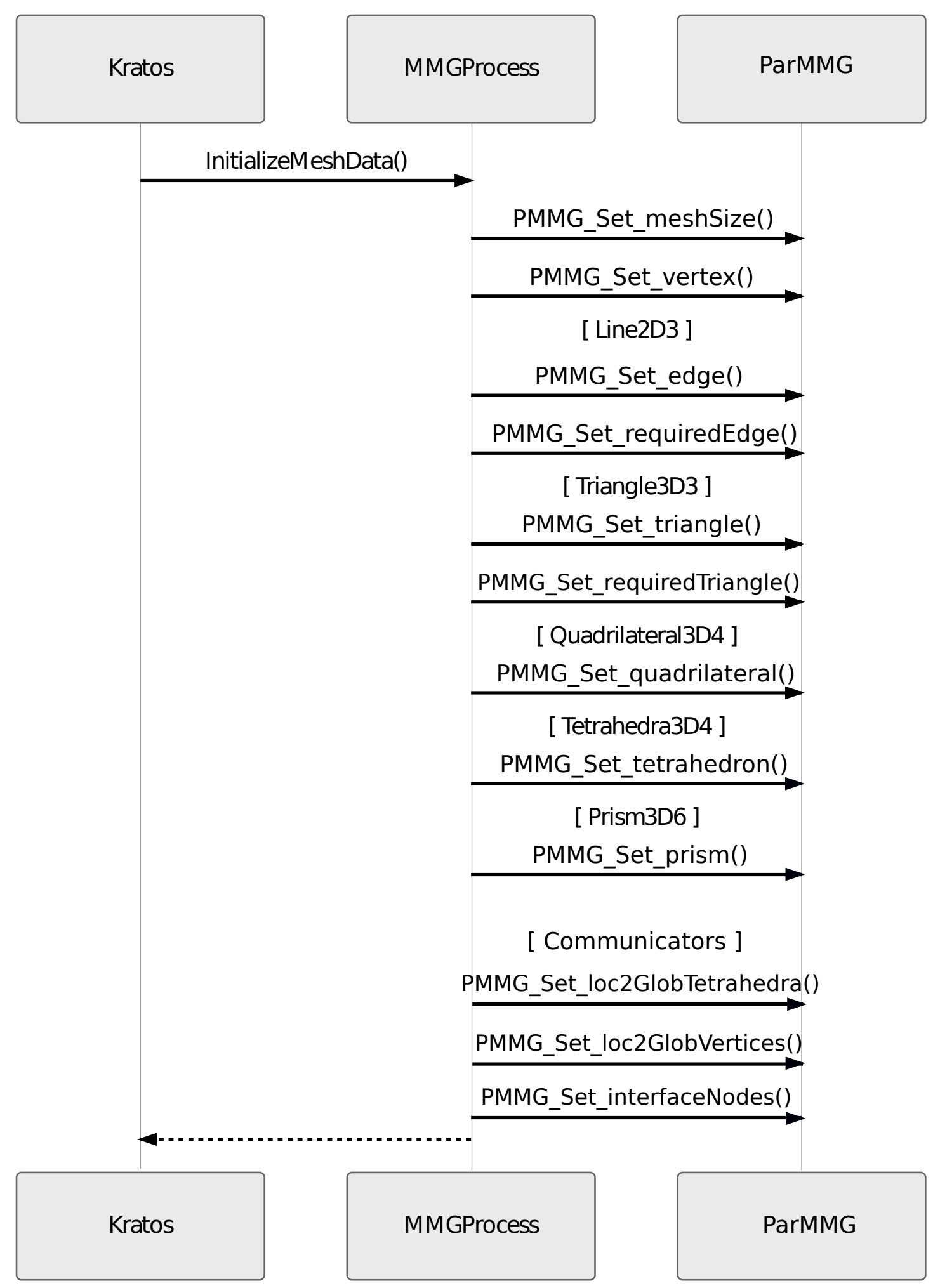

Figure 10: ParMMGProcess Initialize Mesh Data 


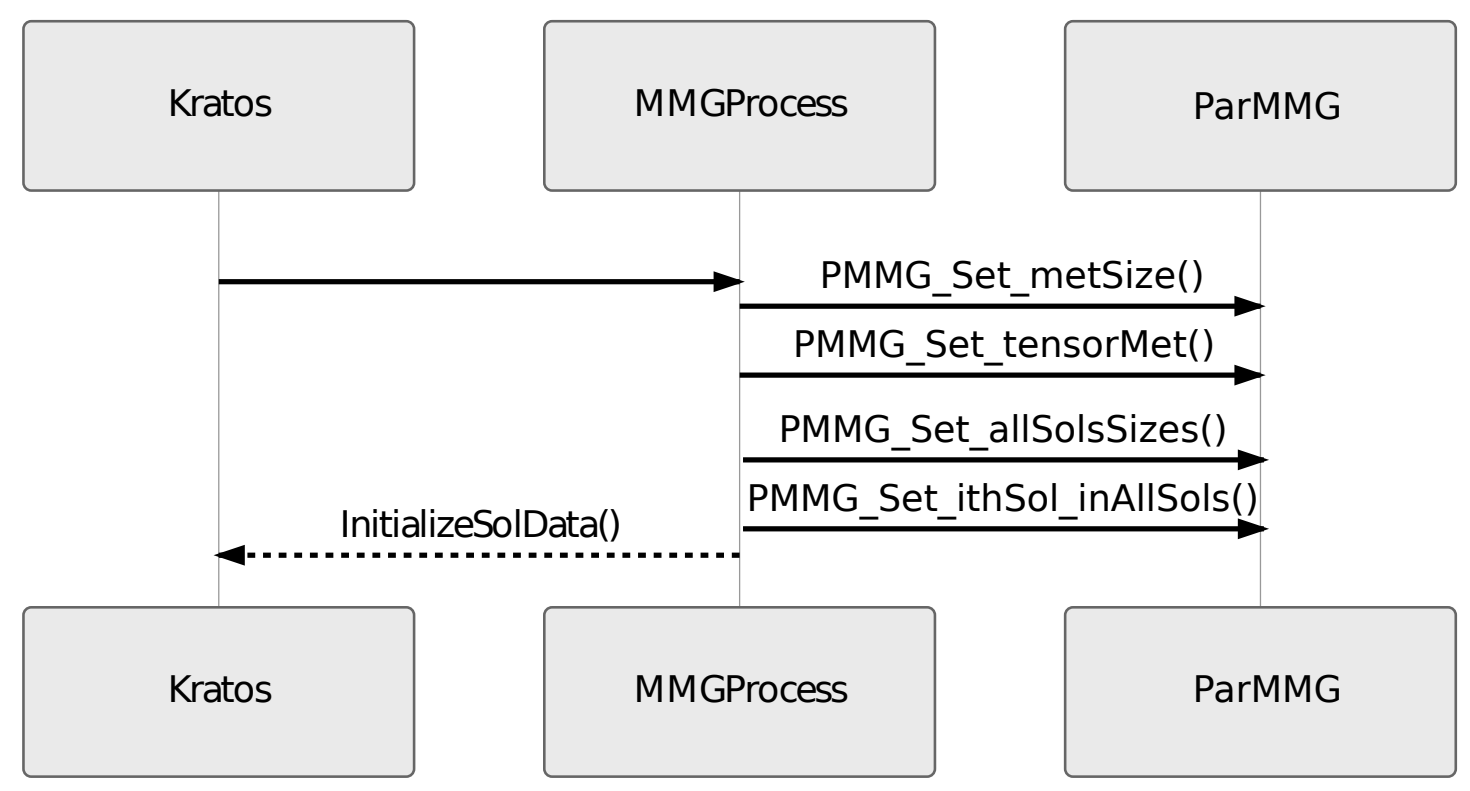

Figure 11: ParMMGProcess Initialize Metric Data

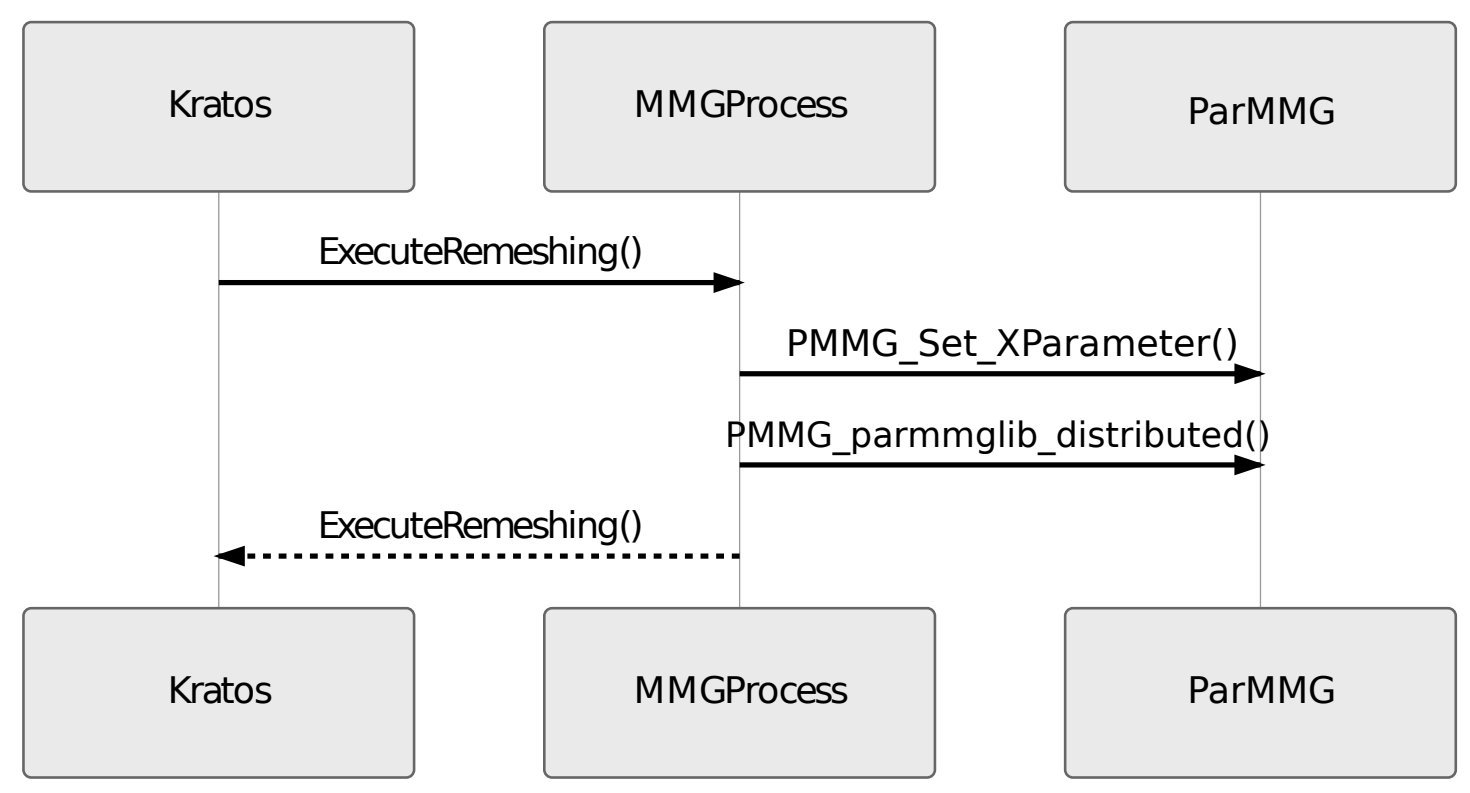

Figure 12: ParMMGProcess Remesh 


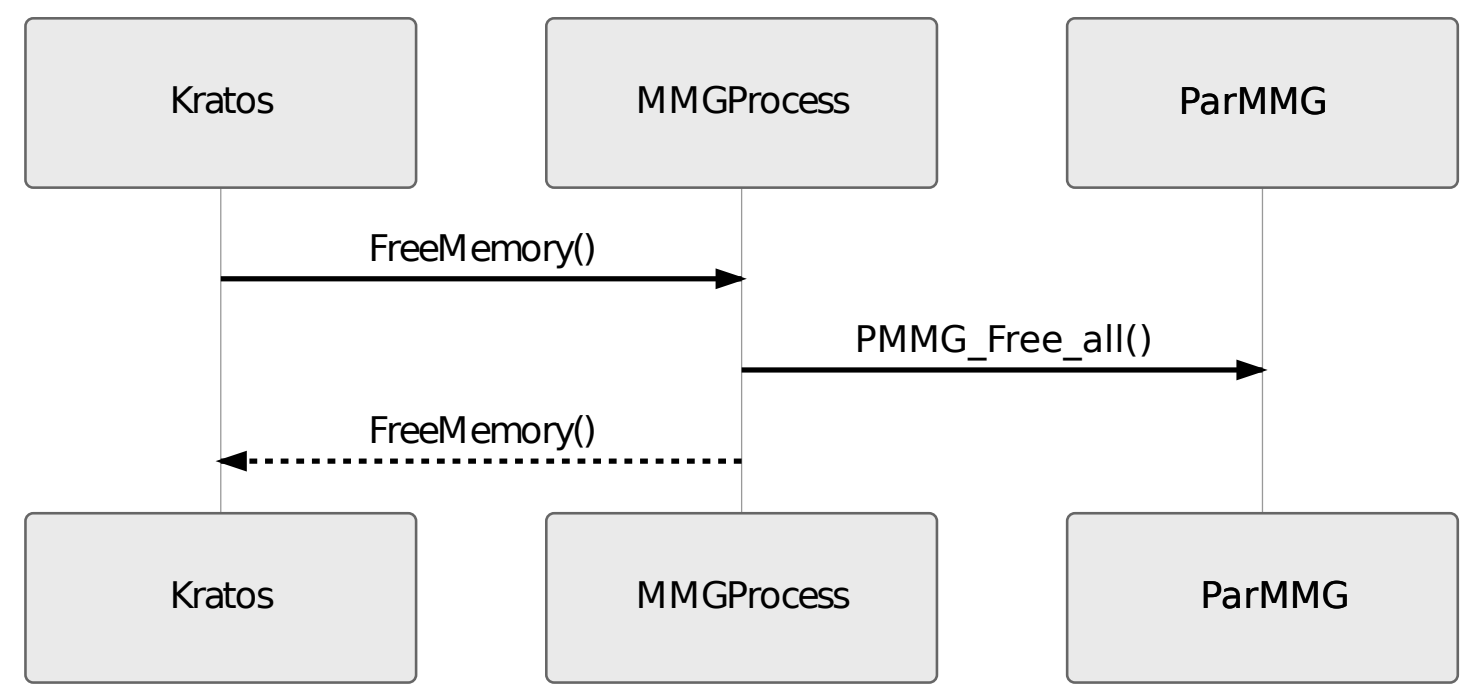

Figure 13: ParMMGProcess Finalize 